\title{
La figura del ciberbardo y la experiencia interactiva del usuario en el diseño videolúdico: L.A. Noire
}

The figure of the cyberbard and the interactive user experience in videoludic design: L.A. Noire

Jesús Albarrán Ligero, Universidad de Sevilla - jesalblig@gmail.com

\section{Resumen}

El objetivo de este artículo es ubicar la narrativa interactiva y el videojuego en sus inicios académicos y reflexionar sobre la figura del diseñador de la experiencia interactiva prefigurando la tensión entre la noción arquitectural y la experiencia interactiva del usuario. Partiendo de los conceptos de ciberbardo y Lector Modelo el artículo se centra en analizar algunos de los supuestos de la gameplay y la estética del videojuego L.A Noire (Team Bondi, 2011).

\section{Palabras clave}

Narrativa interactiva, videojuego, ciberbardo L.A Noire, gameplay.

\section{Abstract}

The aim of this article is to place the interactive narrative and videogame at the begining of their academic studies and to reflect about the interactive experience designer figure, looking at the tension between that architectural notion and the user interactive experience. Starting from the concepts of cyberbard and Model Reader, the article focuses on the analysis of the gameplay and aesthetics of L.A Noire videogame (Team Bondi, 2011).

\section{Keywords}

Interactive narrative, videogame, cyberbard, L.A. Noire, gameplay.

\section{Sumario}

1. Introducción. El legado narrativo-mediático: la narrativa digital interactiva. 2. Supuestos narrativos en el estudio del videojuego. 3. El Modelo Circular de Gameplay: una aproximación al usuario. 4. El lector Modelo en la experiencia videolúdica. 5. L. A. Noire: Shakespeare y la investigación sosegada. 6. Conclusiones. 7. Bibliografía. 


\section{Introducción. El legado narrativo-mediático: la narrativa digital interactiva}

Dentro aquellas dinámicas de lo virtual y la inmediatez que caracteriza a la era de la información, la narrativa interactiva y el videojuego, quizá por este componente lúdico de interacción, han sido objeto de estudio - a veces de manera tangencial— de diferentes disciplinas académicas desde el exponencial desarrollo tecnológico de los años setenta-ochenta. Desde los estudios que apuntaron el valor del juego como evento significativo para la vida cultural del hombre en el primer cuarto de siglo1 - Huizinga (2007), Caillois (1986)_, pasando por las consideraciones filosóficas de Wittgenstein (2004) en el lenguaje o el tratamiento del juego de Gadamer (1991, 2011) en torno a su reflexión con el arte; el juego como valor de interacción junto con la narrativa digital comenzaron a aclimatarse al espacio académico durante los años ochenta en paralelo a aquella domesticación el uso de los primeros ordenadores, videojuegos y experiencias interactivas digitales. Estudios desde la psicología como las aportaciones de David Loftus en Mind at Play. The Psychology of Videogames (1984), investigaban los efectos cognitivos de los usuarios. Pero en su estudio, este primer acercamiento al medio interactivo desde la psicología, equiparaba las nuevas formas interactivas y la narrativa virtual a medios de contenidos narrativos bien asentados durante la segunda mitad del siglo XX como la televisión y la radio. En esta década -dejando atrás fanzines y revistas amateurs de aficionados a esta nueva narrativa interactiva - fue donde comenzaron los primeros estudios específicos sobre videojuegos y narrativas interactivas desde una perspectiva específica. En 1982, Chris Crawford definía "una forma de arte nueva, y de momento poco desarrollada con grandes posibilidades para los diseñadores y los usuarios" (Crawford, 1982: 11) en su libro The Art of Computer Game Design - quizás el primer libro dedicado explícitamente a la teoría y al diseño del videojuego-. Sería en los años noventa cuando se desarrollarían teorías de peso que se dedicaran - a veces de manera tangencial- al estudio específico de "lo interactivo".

El estudio de la narrativa interactiva como texto o "maquina" de expresión dramática para entendernos a nosotros mismos y a nuestro entorno comenzó a forjarse a finales del siglo pasado y a comienzos del nuevo. Diferentes académicos han revisado conceptos narrativos procedentes de otras disciplinas y su idoneidad a la hora de estudiar los nuevos medios narrativos digitales, sobre todo desde la teoría literaria -Gerald Prince, Gerard Gennette-. Sin embargo, sus definiciones, apegadas todavía al legado narrativo mediático, no se ajustaban al nuevo fenómeno y obviaban las peculiaridades interactivas de las nuevas formas digitales. Debido a la ausencia de un campo terminológico y conceptual, no fue extraño que desde la semiótica —como disciplina de gran tradición occidental en la búsqueda del sentido - y la narrativa literaria y fílmica se propiciara un primer acercamiento académico a estas nuevas formas narrativas.

El enfoque postestructutarlista -Derrida (1982), Barthes (1973, 1974, 1977, 1979), Umberto Eco (1984, 1989), Braudillard (1983, 1987, 1993)—y su visión de la literatura como libre de la producción de su autor - y la potenciación en la interpretación del texto por el receptor-, se acercaba en paralelo a la noción decisiva del usuario en los laberintos interactivos del hipertexto tan reconocibles en la emergencia de la narrativa interactiva desde finales de los ochenta y principios de los noventa; puesto que el usuario podía elegir la senda de la historia que más gustase y aventurarse a la exploración narrativa previamente estructurada por el programador. Esta perspectiva supuso de alguna manera la validación de la vertiente postestructuralista y su adecuación en el estudio de los nuevos medios digitales para algunos teóricos -el gran impulsor del hipertexto, George Landow (1997) o Michael Joyce (1995)— (Koenitz, 2015: 93).

Nuevas trayectorias de estudio se han trazado en el estudio de la narrativa interactiva desde que Brenda Laurel abordara la narrativa digital en su influyente libro Computer as Theater (1991) utilizando la poética de Aristóteles para analizar y explicar el drama interactivo. Desde mediados de los noventa, a menudo se ha recurrido al concepto de "narrativa emergente" como síntesis de la autonomía en el jugador -ludólogos- y las aspiraciones narrativas y argumentales —narratólogos - en el medio digital. El término fue acuñado en 1995 por el investigador Tinsley Gaylean:

Nosotros siempre creamos narrativas, al margen de nuestras actividades diarias para ayudarnos a comprender, recordar, categorizar y compartir experiencias. Es esta habilidad la que muchos sistemas interactivos explotan. Ellos nos proporcionan entornos para explorar. Combinando los elementos de ese espacio con nuestros objetivos, permiten emerger la narrativa. Si cualquier estructura narrativa (o historia) emerge es producto de nuestra interacción y objetivos cuando nosotros navegamos la experiencia. Yo llamo a esto narrativa emergente (1995: 27).

Recuperando el concepto de Gaylean, Richard Walsh, reconoce la complicación de esta "narrativa emergente" cuando se aplica al entorno digital, donde reflexiona sobre lo narrativo a nivel cognitivo y como estructura de pensamiento ligada a la temporalidad y a la imaginación, concediendo equilibrio entre la figura del diseñador del juego — el "ciberbardo" de Murray, como veremos más adelante - y las reglas de juego:

Probablemente, la imaginación narrativa siempre comporta una negociación continua entre la representación discursiva del autor y las limitaciones necesarias dentro de un sistema de representación en el cual el acto discursivo alcanza sentido narrativo (2011: 83).

Cómo se llevan a cabo estas negociaciones tiene que ver con el objetivo y las actitudes del jugador; en resumen, con la experiencia de juego. La propia progresión en los estudios de los medios digitales ha demostrado que este legado narrativo se muestra obsoleto e insuficiente en su adecuación y aplicación a unas nuevas formas narrativas interactivas que han surgido en los últimos treinta años y que parece reclaman con inminente necesidad nuevos marcos teóricos y perspectivas que amplíen las dimensiones del estudio.

\section{Supuestos narrativos en el estudio del videojuego}

Reconduciendo este ánimo de Brenda Laurel a la hora de explorar los nuevos medios digitales, dos obras de referencia publicadas en el mismo año 1997 marcarían tanto el impulso en el inicio del estudio de los videojuegos y la narrativa interactiva como la escisión o polarización aparente en dos perspectivas de estudio: Hamlet on the Holodeck (1999) de Janet H. Murray y Cibertext: Perspectives on ergodic literature (1997) de Espen Aarseth.

\footnotetext{
${ }^{1}$ Y cuyo núcleo ha sido muy utilizado como base y punto de partida en los Games Studies.
} 
Dejando a un lado las consideraciones sobre si realmente existió (o existe) una verdadera contraposición o confrontación entre narratólogos² -académicos que siguiendo la estela de Murray estudian el juego como un medio de potencialidades narrativas - y ludólogos - partidarios de Aarseth, que reconocen esta nueva experiencia interactiva como una "maquina" o texto creador de significados cuyo análisis tiende a favorecer el estudio de sus estructuras, mecánicas y reglas-, lo cierto es que esta nueva interacción digital entendida integralmente como "maquina" o "artefacto" ecléctico que nos sirve para reconocernos a nosotros mismos y a nuestro entorno conforma una noción más rica e integradora para el estudio del videojuego incluso a nivel narrativo. Para Aarseth, este cibertexto, es un espacio en el que es posible perderse y explorar "no metafóricamente sino respecto a las estructuras topológicas de la maquinaria textual” (Aarseth, 1997: 4-5). Y considera diferencias fundamentales entre narración y juego, argumentando que considerar ambas realidades análogas significa despreciar características esenciales de cada una de ellas.

Aunque el significado de "texto" o "maquina" es más cercano al trabajo "filológico (u observable) que a la galaxia de significados postestructural o metafísico" (Aarseth, 1997: 20). Es evidente que el significado de "texto" en Aarseth se refiere a ambas realidades, utilizando la palabra para designar a un amplio rango de fenómenos - desde poemas interactivos, pasando por bases de datos hasta videojuegos_-donde participen los tres actores de la máquina: operador (usuario, en el caso de los videojuegos), señal verbal (todo tipo de señal verbal, comunicativa) y medio (videojuego: pantalla y pad 0 algún dispositivo de input -teclado, gafas virtuales...).

Murray explica en Hamlet on the Holodeck las cuatro propiedades que considera esenciales de los entornos digitales: los entornos digitales son secuenciales, participativos, espaciales y enciclopédicos (1999: 83). Y esta participación conlleva en su núcleo el factor interactivo del medio y la consideración de contar con el usuario cuando se programa (1999: 90). En este sentido, el concepto de "ciberbardo" de Murray como figura de autor que asiste a todo el proceso estético y configurativo —en definitiva, a toda la experiencia canónica interactiva-, emerge como figura esencial en la planificación arquitectural del juego:

Los ciberautores controlarán las técnicas de autoría de hipertextos, que les permitirán establecer no sólo las palabras e imágenes de la historia, sino también las reglas por las que se regirán esas palabras e imágenes (...) [Los usuarios] aceptarán que su actuación forma parte de la experiencia estética (1999: 284).

Desde la aparición de la ludología, auspiciada desde las páginas de Games Studies ${ }^{3}$, se ha explorado la narrativa como elemento esencial en el debate sobre el videojuego - Marie-Laure Ryan (2001), Jesper Jull (2001), Eskelinen (2001) - . Como bien había apuntado el antropólogo y filósofo francés Paul Ricoeur (1991), una de las definiciones de narrativa más extendida es aquella que proclama la narrativa como manera de organización del conocimiento en estructuras llenas de significado que nos ayudan a entender el mundo. Si esto es así, cuando aparecen nuevos géneros que abran nuevas maneras de experimentar esta manera de organización, parecería obvio y necesario un nuevo marco experimental y relacional en el estudio del objeto. Koenitz concede esta noción generativa al planteamiento de Murray con respecto a las nuevas narrativas interactivas:

Esto significa que como práctica propone desarrollar nuevos y distintos formatos y géneros narrativos, no meras nuevas versiones de formatos establecidos tales como la novela, la película o el drama en el escenario. En consecuencia, Janet Murray ha identificado "Ia invención y el refinamiento" de las convenciones del diseño como área de atención para la investigación práctica en los medios digitales expresivos, para así evitar el daño de "tentativas no productivas de aplicar convenciones heredadas a la nueva infraestructura digital" (Koenitz, 2015: 52).

Si como apunta Murray existen nuevos formatos narrativos que están revolucionando - desde los años ochenta — la manera de entender el mundo a nivel de participación e implicación en las historias, es obvio que la terminología y la herencia, el legado de los medios no se haya adecuado a las nuevas exigencias y formas digitales, ya que la emergencia del desarrollo tecnológico propicia a principios del siglo XXI una revitalización del debate terminológico y conceptual entorno a la noción de narrativa. Es necesario atender a diferentes acepciones para esbozar qué se entiende o se ha entendido tradicionalmente por narrativa en el ámbito de las ciencias sociales y su extrapolación al estudio del videojuego y la narrativa interactiva (extraído de Jull, 2005: 156):

1. Narrativa como presentación de un número de eventos. El significado literal y tradicional de "narrativa" o "cuentacuentos" (Bordwell 1985; Chatman, 1978).

2. Narrativa como una secuencia fija y predeterminada de eventos (Brook, 1992).

3. Narrativa como un específico tipo de secuencia de eventos (Prince, 1987).

4. Narrativa como un específico tipo de tema -humano o de entidades antropomórficas (Grodal, 1997).

5. En un sentido más amplio, narrativa como cualquier tipo de escenario o mundo ficcional: transmedia, etc (Jenkins, 2003).

6. Narrativa como la manera que damos sentido al mundo (Schank y Abelson, 1977).

Como bien expresa Jull, muchos de los escritores críticos con la aplicación de la teoría narrativa en los nuevos medios digitales han focalizado su atención en las tres primeras nociones de narrativa (identificadas con el estudio de medios), mientras que los investigadores que apuestan por la integración teórica entre la noción lúdica (experiencia) y su vertiente narrativa en el estudio del videojuego suelen acogerse a la quinta y sexta acepción de narrativa, más cercanas al amplio campo de la Psicología social, la ciencia cognitiva o la Filosofía.

En la reciente actualidad, muchos estudios y análisis sobre la figura del autor digital aún consideran que se debe cultivar las potencialidades del "ciberbardo" y aplicar una pedagogía para las nuevas generaciones de creadores digitales, estimulando la creatividad y abriendo camino para la

2 Es pertinente establecer aquí una diferenciación terminológica; ya que la Narratología en los estudios académicos semióticos se conoce como la disciplina a la que compete el estudio estructural de los relatos. Con afán esclarecedor, el teórico Gonzalo Frasca (1999) —siguiendo a Mateas (2002) - ha propuesto diferenciar entre narrativistas, investigadores que utilizan la Teoría Literaria como base para construir una posterior teoría centrada en el medio interactivo y narratólogos, académicos que trabajan desde postulados narrativos independientemente del medio objeto de investigación; estableciendo así el objeto de estudio como diferencia esencial.

3 Primera revista académica específica sobre el videojuego fundada por Espen Aarseth en 2001. Fue este año cuando el mismo Aarseth proclamó "el año del videojuego" debido a la cantidad de conferencias, másteres y cursos académicos que comenzaban a interesarse por el objeto de estudio del videojuego desde finales del siglo pasado. 
creación de nuevas experiencias interactivas donde la teoría narrativa y la práctica interactiva se encuentran permanentemente conectadas en un feedback donde las consideraciones de Aarseth siguen resultando influyentes. Existen teóricos que han abrazado nuevos términos con afán integrador a la hora de hablar de este loop interactivo en el medio digital.

Recientemente, el investigador utrechtense Koenitz siguiendo las tesis de Murray apunta que esta iteración que nos lleva de lo narrativo a lo interactivo propicia una distinción, una doble vertiente extraída e identificada de alguna manera del legado mediático narrativo: la pura narrativa estructurada desde el autor - que él reconoce como el antiguo plot o discurso—; la "manifestación material fija" a la vez que una parte "flexible" de la narrativa que se construye fácticamente a través de la instanciación ${ }^{4}$ y resulta ser la materia prima de esta nueva narrativa interactiva.

Un marco teórico para analizar esta nueva narrativa lo propone su concepto de protohistoria: "(La protohistoria) describe el interfaz interactivo, y en este sentido, encarna el propósito artístico que permite un proceso participativo de instanciación resultante de la materialización o realización de las narrativas potenciales" (2015: 99). En este sentido, la protohistoria comprendería todo lo fijado en el formato del videojuego que puede ser potencialmente jugable y que contribuya así a la realización de su narrativa inextricable, ya que esta es concebida por Koenitz de alguna manera como la experiencia de juego en sí.

Para Koenitz, no existe división entre estructura y contenido dentro de las narrativas potenciales que propone la narrativa flexible digital, por lo que valida las tesis de Aarseth en el sentido de considerar a estas experiencias narrativas digitales como un "texto" en sentido amplio por donde el usuario puede "transitar" literalmente y cuyas estructuras cobran sentido en tanto en cuanto son experimentadas por el usuario (Aarseth, 1997: 1) -Koenitz lo llama instanciación, el proceso de participación activa del jugador-. Por otro lado, otorga un gran protagonismo al ciberbardo y a la construcción cerrada y canónica de una historia en sí misma a través de los "vectores narrativos". Sin embargo, parece que su modelo se reconoce más ubicado o se aplica con especial comodidad en experiencias narrativas apegadas a la literatura 0 al experimento literario digital, donde la exploración se encuentra gradualmente limitada por las reglas de la experiencia interactiva y sin embargo concede al usuario la ilusión de flexibilizar y co-escribir la historia: una experiencia cercana a lo que se conoce en el mundo anglosajón como Interactive storytelling. Es significativo que el modelo de Koenitz, una de las últimas aportaciones en teoría narrativa digital, se encuentre cómodo entre las primerizas obras de narrativa interactiva como el hipertexto o las películas interactivas. Parece que mientras más cerca se encuentra la experiencia digital de una jugabilidad compleja o de un proceso de instanciación que se aleje de las formas tradicionales de contar menos reconocibles o difusos se muestran estos vectores narrativos, 0 al menos más dificultad encontramos en identificarlos y desgajarlos de la mera narrativa proposicional.

La revisiones de Koenitz en una teoría holística de las nuevas narrativas propuestas por Dubbelman Teun, Knollel Noam, \& Roth Christian (2015) designan dos grandes constructos en los que el usuario se ve envuelto: el primero es la Protohistoria — como una manera de fijar los acontecimientos, el hardware y software que ocurren en el juego - y el segundo es el Modelo de Interacción. El Modelo de Interacción representa la consideración del entorno digital como una manera de "estructurar la actividad encarnada por el usuario" (Dubbelman, Knollel, Koenitz y Christian, 2015: 55), la lógica de su comportamiento inscrito en el sistema -y por tanto, la fijación de unos límites en la experiencia interactiva-. Ambos constructos representan lo que Bogost ha reclamado como "el discurso configurativo" o retórica procedural en el videojuego que alcanza su forma más depurada en los Persuasive Games (2007) revitalizando la esencialidad de la estructura y estrategia reglada prefijada por el ciberbardo en la experiencia interactiva. Pero desde el mismo Bogost, se intuye una inadecuación que hace imposible la proyección unilateral de una narrativa o estrategia discursiva o de juego que precipite estos presupuestos discursivos que se desprenden de las decisiones autorales. En su concepción de los juegos, Bogost entiende que existe una franja de desconocimiento o desajuste que impide la concreción semántica de la experiencia que prefija el autor. Con la noción de Simulation fever, Bogost explora las reacciones negativas al texto. Esta brecha muestra un espacio intermedio entre la resignación de la simulación (Simulation resignation) - "la aceptación ciega de los límites resultantes de la simulación, porque el sistema no permite ningún otro modelo de sistema de referencia" y la negación de simulación (Simulation denial) -el rechazo de la simulación porque implica una representación simplificada del sistema fuente- . Para Bogost, "simulación" supone "la brecha existente entre la representación basada en reglas de un sistema fuente y de la subjetividad del usuario" (Bogost, 2006: 107). La franja existente entre el sistema de juego prefijado por el ciberbardo o el diseñador de juego y la subjetividad del individuo - simulation gap-, un espacio que en los últimos años han intentado copar estudios generativos que han dado lugar a interesantes propuestas como las dinámicas cognitivas que experimenta el usuario a través de procesos abductivos (Navarrete, Pérez, \& Pérez, 2014).

\section{El Modelo Circular de Gameplay: una aproximación al usuario}

Las propuestas teóricas y metodológicas basadas en el proceso que experimenta el usuario parece un territorio apenas explorado por el punto de fijeza moral que otorga Miguel Sicart en Ethics of Computer Games (2009). Sin embargo, jugar o establecer un tipo de relación con la obra interactiva es un proceso circular que va más allá de la planificación autoral. Durante el tiempo de juego o "experiencia" narrativa nos encontramos inmersos en un proceso que algunos teóricos han calificado como cercano a la autonarración5; un feed-back constante de información (decodificación), estrategia y codificación complejo de analizar, estructurar y cartografiar debido a la propia naturaleza lúdica del medio interactivo. Desde la lógica de los estudios sobre videojuegos, el Modelo Circular de Gameplay propuesto por Heaton (2006), donde la decisión del jugador se encuentra en el centro de este loop, parece pertinente para sugerir la naturaleza dúctil del medio ya que describe al mismo tiempo un proceso cognitivo y mecánico del usuario. Tomando como modelo los supuestos de Heaton, Bernard Perron (2006) enriquecerá el modelo de la experiencia de juego con el Modelo del Círculo Heurístico de la Gameplay.

\footnotetext{
${ }^{4}$ El concepto más cercano que se observa para esta "manifestación material fija" en el videojuego lo protagonizan las secuencias narrativas puras, aquellas en las que el jugador no interactúa, no ejerce sus energías en transformar "existentes" y en activar "eventos" - utilizando la terminología de Seymour Chatman (1978) - y sigue el flujo secuencial de la narrativa, como parte de la audiencia. (Durante las llamadas CGI o secuencias de "animación por ordenador" tan populares en los años noventa). Mientras que la "narrativa flexible" es aquella predispuesta y codificada en la propia partida: la experiencia de juego interactiva.

5 Iván Martínez (2016) se ha aventurado a calificar a este loop, o feedback narrativo interactivo como Retronarratividad. En los últimos años, algunos teóricos españoles están versando o han versado sus Tesis sobre narrativa y videojuego acercándose al objeto sobre todo desde su estética.
} 
El proceso cognitivo ascendente (Botton-up process) se encarga de procesar la información audiovisual, de extraer información este proceso se encuadra en la dinámica cognitiva desde lo particular a lo general- (desorientación o disolución de esquemas, por lo que el usuario prestará más atención a la información en pantalla: al audio y al audiovisual), mientras que el proceso verticalista (Top-down process) se encuentra dominado por el plano del concepto y del esquema ${ }^{6}$ - por lo que responde al movimiento cognitivo desde lo general a lo particular - (expectación del usuario generada por esquemas reconocibles). Los dos procedimientos suelen trabajar juntos: "Once you have analysed the situation, you can decide on a procedure to maximize your actions (top-down process). But you also have to rely on the data (bottom-up process), because the game creates its own states" (Perron, 2006: 64).

\section{El Lector Modelo en la experiencia videolúdica}

Estas decisiones arquitecturales que toman los desarrolladores del juego no sólo afectan a su vertiente configurativa. Como ya apuntara Pérez Latorre (2012), particularmente interesante para el estudio de la estética y de la gameplay en el videojuego son aportaciones de la semiótica como la teoría del Lector Modelo de Umberto Eco formulada en su ensayo Lector in Fabula, donde la enunciación de un relato no implica solo la creación de un

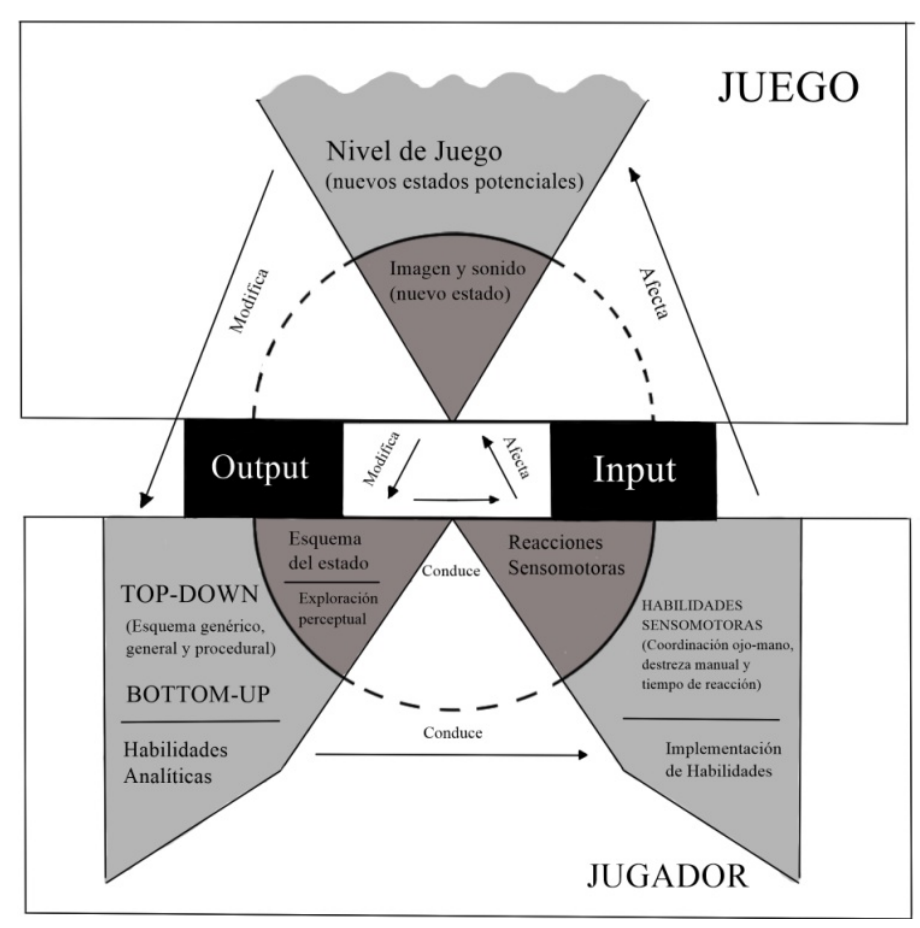
texto, sino también la elaboración de una experiencia prefijada del lector relativamente presupuesta; es decir, la disposición de una estrategia textual:

Un texto es un producto cuya suerte interpretativa debe formar parte de su propio mecanismo generativo: generar un texto significa aplicar una estrategia que incluye los movimientos de otros (...). Para organizar su estrategia textual el autor debe referirse a una serie de competencias capaces de dar contenido a las expresiones. Debe suponer que el conjunto de competencias a que se refiere es el mismo al que se refiere su lector. Por consiguiente, deberá prever un Lector Modelo capaz de cooperar en la actualización textual de la manera prevista por él, y de moverse interpretativamente, igual que él se ha movido generativamente (Eco, 1993: 79-80).

En este sentido, H. Murray también señala una estrategia similar en el diseño de entornos virtuales, aunque sea a nivel de reconducir el background tanto en la dimensión narrativa como de gameplay y programación de un determinado perfil de jugador al utilizar un conjunto "restringido de convenciones literarias y lúdicas":

El primer paso para hacer un mundo narrativo atractivo es considerar al usuario cuando se programa (...). El entorno fantástico le da al usuario un papel familiar y hace posible que los programadores anticipen las reacciones del jugador. Al utilizar estas convenciones literarias y lúdicas para limitar las acciones del jugador a un conjunto restringido de órdenes dramáticamente apropiadas, los diseñadores pudieron concentrar sus poderes imaginativos en hacer que el mundo virtual respondiese adecuadamente a cualquier combinación de estas órdenes (1999: 90-91).

El concepto de Lector Modelo dentro de la lógica de la arquitectura del medio interactivo resulta muy útil a la hora de interpretar textos y fijar un punto de anclaje en el usuario, tanto en el estudio formal relativo a la gameplay como al expresivo más cercano a la disposición creativa y ficcional que potencia su dimensión narrativa, la noción "no ergódica" a la que hacía referencia Skelinen (2001). Esta estrategia interpretativa, se revela así tanto en la construcción de una gameplay coherente y ajustada a un tipo de jugador como en la estética, —-dimensión narrativa y mundo ficcionaldel videojuego - ya que ambas se encuentran íntimamente ligadas-.

\section{L.A. Noire: Shakespeare y la investigación sosegada}

L.A Noire (Team Bondi, 2011) es un juego a medio camino entre un sandbox —videojuego no lineal— y una aventura gráfica donde el usuario encarna al detective Cole Phelps. El juego se estructura en diferentes casos donde el jugador debe encontrar e interpretar pistas, interrogar a sospechosos/testigos y moverse libremente por la inmensa ciudad de Los Ángeles ambientada en los años cuarenta como mapeado totalizador de las diferentes fases/casos. Durante una misión, Cole encuentra lo que parece una cabeza reducida africana en un coche despeñado, cuando un colega se la tiende para que la examine como posible prueba del accidente, Phelps exclama sin razón aparente y con ademanes excesivamente dramáticos: "iAh, pobre Yorick!, ¡Yo lo conocí Horacio!"; para, a continuación, cambiar su registro y adoptar una posición seria y profesional ante el caso: "¿Esta cabeza es de verdad?".

Ah, poor Yorick! Here hung those lips that I have kissed I know not how oft. Where be your gibes now? Your gambols? Your songs? Your flashes of merriment, that were wont to set the table on a roar? (Shakespeare, 2003: 231).

\footnotetext{
${ }^{6}$ El término "esquema" adquiere en el círculo heurístico de Perron la resonancia de aquella definición acuñada por Neisser: "Un esquema es la porción del círculo perceptual completo que es interna al que percibe, modificable por la experiencia y, de algún modo, específica para lo que está siendo percibido. El esquema acepta información cuando se encuentra disponible en la superficie sensorial y es transformado por esa misma información, esto conduce a movimientos y a actividades exploradoras que hace que más información se encuentre disponible, por la que es más tarde modificado de nuevo" (1976: 54).
} 
Este fragmento pertenece al acto 5, escena 1 de la obra de William Shakespeare Hamlet, donde el príncipe holandés encuentra el cráneo de Yorick, el bufón de la corte ahora muerto en el cementerio, y que da lugar a uno de los monólogos más populares de la historia del teatro sobre la ironía y el azar en el destino de los mortales. La elección del comentario jocoso de Phelps, pero sobre todo el hecho de que no exista ningún recurso que reformule, clarifique o ubique el locuaz comentario del detective -que ninguno de los personajes presentes apunte a qué se refiere o lo sugiera: los demás personajes ignoran el comentario— es una clara marca de enunciación narrativa y una llamada a la complicidad del lector/usuario. Sin dejar de ser un gag prescindible para el desarrollo y el disfrute de la gameplay, su propia enunciación es indicativo de una tendencia en el enfoque de su narrativa, incluso de su propio diseño de juego: el jugador/lector modelo de L.A Noire, está provisto —o debería— de cierta sensibilidad literaria 0 , como mínimo, de un bagaje cultural heterogéneo de base —-inematográfico o escénico- que le permita identificar la referencia intertextual de Yorick.

Esta concepción del usuario cultural en L.A Noire, está plasmada en la propia estética del juego. La elección del actor Aaron Staton para el bodycapture como actor ocasional en series de televisión de época y afán detectivesca con significativos recursos metafóricos, personajes complejos más cercanos al teatro y amplias referencias literarias y culturales que enmarcan las tramas -inevitables resonancias con series que ha protagonizado anteriormente: Mad Men (AMC, 2009), Law and Order: Special Victims (NBC, 1999) - no parece ser casual e invita a enfrentar al juego desde su inicio con una complejidad y densidad argumental que se extiende de manera icónica hasta su personaje principal —que, en última instancia, encarna el jugador-. Su banda sonora influenciada por el jazz clásico de los años cuarenta más acorde con el espíritu sosegado de dedicación que el Swing o el Bebbop de igual vigencia y éxito por aquellos años tutela un afán introspectivo. En el plano de la gameplay, L.A Noire se acerca a un modelo de juego donde se premia el ingenio, la sagacidad e intuición a la hora de encontrar pistas, interpretarlas y descifrar los gestos faciales de los testigos y sospechosos.

Los casos se estructuran en tres diferentes eventos que pueden presentarse de manera intermitente o desordenada: 1. Búsqueda e interpretación de pruebas. 2. Interrogatorio de testigos y sospechosos: valoración, indagación y estudio de sus respuestas faciales. 3 . Algunos eventos de acción (reyertas y persecuciones). Todo enmarcado dentro de la experiencia sandbox, con la posibilidad de exploración a los eventos accesorios o no esenciales para completar la aventura de Phepls: 4. Misiones especiales; más sujetas a eventos de acción que dinamicen la trama. Aunque el sistema de juego pudiera sugerir una dinámica similar a grandes éxitos del sandbox como Grand Theft Auto III (Rockstar, 2001) o Mafia III (Hangar 13, 2016), la dinámica de L.A Noire se presenta como una narrativa muy lineal en cuanto el usuario comenza las misiones esenciales y se basa estéticamente en tópicos cinematográficos y literarios de la época.

La dinámica de juego de L.A Noire presenta una potenciación de valores basados en la inteligencia, la comprensión y la reflexión sosegada y su narrativa apunta de igual modo a una alta sensibilidad en el jugador; una sensibilidad artística y un bagaje cultural en el usuario. El nivel de acción es reducido - reservados en la mayor parte al punto 4; las misiones secundarias-con respecto a los momentos de investigación e inmersión criminal. En esta dinámica de gameplay reverbera este pensamiento sosegado y unidireccional: hay una única respuesta correcta: ensayo y error. Todo sugiere que el jugador modelo de L.A Noire debería contar con cierta sensibilidad humanista; el lector de novelas policiacas, amante del cine; paciente y reflexivo. La tonalidad del juego se encuentra marcada por el molde de un usuario que encaja con su gameplay y su estética.

\section{Conclusiones}

La asunción de un usuario implícito en el diseño de juego es útil no sólo a nivel de la planificación de los límites de juego, sino que también a nivel estético. El trabajo del ciberbardo a la hora de crear una arquitectura del videojuego trabajando con la noción implícita del usuario transforma la obra interactiva en una experiencia más madura e interesante a la hora de potenciar tonalidades caras al jugador; pero no hay que olvidar que el juego es ante todo "una experiencia" y como experiencia, ésta es limitada sólo por el usuario. A la hora de enfrentarse al videojuego y a la narrativa interactiva como "artefacto" se deben de concebir ante todo como desencadenantes de una experiencia polivalente, no sólo como un texto prefijado por el autor: este existe de manera arquitectural y a la vez es transitado por el usuario. La manera de crear obras interactivas más interesantes es potenciando la capacidad plástica de la propia gameplay y de su estética. Coincidimos en que el legado narrativo -y terminológico- se ha mostrado históricamente inadecuado a la hora de analizar la impronta interactiva, propiciando un acercamiento al objeto de estudio contagiado por las formas y lógicas de los medios audiovisuales y narrativas tradicionales, pero existen conceptos que revisionados pueden resultar útiles y expandir el análisis de los textos interactivos videolúdicos. Cualquier experiencia interactiva se encuentra unida al proceso circular de gameplay que permite describir la experiencia lúdica acercándose a la riqueza de todo el proceso experimentado por el usuario. Ciertamente, el camino futuro que parecen tomar las nuevas narrativas interactivas se acerca más a la potenciación de la experiencia e inmersión sensorial -eliminación de traductores analógicos, gafas VR (Realidad Virtual) - que a la lógica narrativa en sentido estricto - vagamente apegada a la secuencialidad del plano y al encuadre fílmico-. Esto nos hace reflexionar sobre la dirección que debe tomar la plasticidad de la nueva narrativa digital. El medio digital requiere de nuevos conceptos, expresiones y formas de estudio más flexibles para afrontar los nuevos formatos que quizá deban dar respuesta a cuestiones más inmersivas: "¿cómo interactuar, experimentar, percibir dentro de un entorno de narrativa envolvente?" que al problema de “¿cómo contar?". En este sentido, es tiempo de propuestas interdisciplinares que abran el campo de estudio ante la fijeza de modelos cartográficos y que los diseñadores de videojuegos observen cómo potenciar y moldear el lienzo donde los usuarios despliegan la experiencia.

\section{Bibliografía}

Aarseth, Espen (1997): Cibertext: perspectives on ergodic literature. Baltimore: John Hopkins University Press.

Barthes, Roland (1973): Mythologies (selection from this and others Works). New York: Noonday Press.

Barthes, Roland (1974): S/Z. New York: Hill and Yang.

Barthes, Roland (1977): The death of the author. En: Image, Music, Text. New York: Hill.

Barthes, Roland (1979): From the work to text. En: Harari, J. (ed.) Textual Strategies: Perspectives in Posestructuralist Criticism. Ithaca: Cornell University Press. 
Bogost, lan (2006): Unit Operations. Cambridge: The MIT Press.

Bogost, lan (2008): Persuasive Games: The Expresive Powers of Videogames. Cambridge: The MIT Press.

Bordwell, David (1985): Narration in the fiction film. Wisconsin: University of Wisconsin Press.

Braudillard, J. (1983): Simulations. New York: Semiotext.

Braudillard, J. (1987): The Ecstasy of Communication. New York: Semiotext.

Braudillard, J. (1993): System of Objets. New York: Verso.

Brook, Peter (1992): Reading for the Plot: Desing and Intention in Narrative. New York: Alfred A. Knopf.

Caillois, Roger (1986): Los juegos y los hombres. La máscara y el Vértigo. México: Fondo de cultura económica.

Chatman, Seymour (1978): Story and Discourse: Narrative Structure in fiction and Film. Ithaca, Estados Unidos: Cornell University Press.

Crawford, Crish (1982): The Art of Computer Game Design. http://www-

rohan.sdsu.edu/ stewart/cs583/ACGD ArtComputerGameDesign ChrisCrawford 1982.pdf [Consultado el 13/12/2016].

Derrida, J. (1982): Margings of Philosophy. Chicago, Estados Unidos: University of Chicago Press.

Dubbelman Teun, Knollel Noam, Koenitz Hartmun \& Roth Christian. (2015): All Integrated and Iterative Research Direction for Interactive Digital Narrative. En Frank Nack \& Andrew Gordon (coords.), Interactive Storytelling (pp. 51-60) Springer: Suiza.

Eco, U. (1984): The role of the reader. En: Exploration in Semiotics of Texts. Broomington: Indiana University Press.

Eco, U. (1989): The Open Work. Cambridge: Harvard University Press.

Eco, U. (1993): Lector in fabula. La cooperación interpretativa en el texto narrativo. Barcelona: Editorial Lumen.

Eskelinen, M. (2001): The Gaming Situation. Games studies, 1 (1), 68. http://gamestudies.org/0101/eskelinen/ [Consultado el 21/12/2016].

Foucault, M. (1972): Archaelogy of Knowledge. New York, Estados Unidos: Pantheon.

Foucault, M. (1977): "What is an author?" En: Bouchard, D.F. (ed.) Language, Counter-Memory, Practice. Ithaca, New York, Estados Unidos: Cornell University Press.

Frasca, Gonzalo (1999): Ludology Meets Narratology: Similitude and Differences between (Video) Games and Narrative. http://www.ludology.org/articles/ludology.htm [Consultado el 14/12/2016].

Gadamer, Hans Georg (1991): La actualidad de lo bello. Barcelona, España: Ediciones Paidós/l.C.E-U.A.B.

Gadamer, Hans Georg (2011): Estética y hermeneútica. Madrid, España: Editorial Tecnos.

Gaylean, Tinsley (1995): Narrative Guidance of Interactivity. Tesis Doctoral. Massachusetts, Estados Unidos: MIT Press.

Grodal, Torben (1997): Moving Pictures- A New Theory of Film Genres, Feelings and Cognition. Oxford, Inglaterra: Oxford University Press.

Heaton, Tom (2006): A Circular Model of Gameplay. En Gamasutra.com.

https://www.gamasutra.com/view/feature/130978/a_circular_model_of_gameplay.php [Consultado el 14/12/2017].

Huizinga, Johan (2007): Homo ludens. Barcelona, España: Alianza editorial/Emecé.

Jenkins, Henry (2003): Transmedia Storytelling. https://www.technologyreview.com/s/401760/transmedia-storytelling/ [Consultado el 13/12/2016].

Joyce, M. (1991): Of Two Minds: Hypertext Pedagogy and Poetics. Ann Arbor, Estados Unidos: University of Michigan Press.

Jull, Jesper (2001): Games telling stories?: A brief note on games and narratives. Game Studies, vol. 1 (1) http://www.gamestudies.org/0101/juulgts/ [Consultado el 5/12/2016].

Jull, Jesper (2005): Half-Real: Video Games between Real Rules and Fictional Worlds. Cambridge, Estados Unidos: The MIT Press.

Koenitz, Hartmut (2016): Towards a Specific Theory of Interactive Digital Narrative. En: Frank Nack \& Andrew Gordon (coords.), Interactive Digital Narrative (pp 91-102) New York, Estados Unidos: Routledge.

Landow, G. (1997): Hypertext 2.0. Baltimore, Estados Unidos: John Hopkins University Press.

Latorre, Oliver (2012): El lenguaje videolúdico. Análisis de la significación del videojuego. Barcelona: Laertes. 
Laurel, Brenda (1991): Computer as Theater. Reading, Estados Unidos: Addison-Wesley.

Loftus, G. R. \& Loftus, E. F. (1984): Mind at Play. The Psychology of Videogames. New York, Estados Unidos: Basic Books.

Martínez, Ivan (2016): Análisis de la retronarratividad como fundamento estético del videojuego. (Tesis doctoral). Universidad de Sevilla, Sevilla.

Mateas, Michael (2002): Interactive Drama, Art and Artificial Intelligence. Tesis doctoral, School of Computer Science, Carnegie Mellon University, Pittsburgh: PA.

Murray, J.H. (1999): Hamlet en la holocubierta. Barcelona: Paidós.

Murray, J.H. (2012): Inventing the Medium: Principles of Interaction Design as a Cultural Practice. MIT Press: Cambridge.

Navarrete Jose Luís, Pérez, Jose Patricio \& Pérez, Francisco (2014): El pensamiento abductivo como fundamento ontológico de los videojuegos. ICONO vol. 12. Vol. 2, pp. 416-440. https://idus.us.es/xmlui/bitstream/handle/11441/24719/elpensamientoabductivo.pdf?sequence=1\&isAllowed=y [Consultado el 12/12/2016].

Perron, Bernard (2006): The Heuristic Circle of Gameplay: the Case of Survival Horror. En Santorineos, Manthos (ed). GAMING REALITIES: A challenge for digital culture, pp. 62-69. Atenas: Fournos.

Prince, G. (1987): A Dictionary of Narratology. Lincoln, Estados Unidos: University of Nebraska Press.

Ricoeur, Paul (1991): Narrative Identity. Philosophy Today. vol. 35, 73-81.

Ryan, Marie-Laure (2001): Beyond Myth and Metaphor: The Case of Narrative in Digital Media. Games Studies, vol. 1 (1). http://www.gamestudies.org/0101/ryan/ [Consultado el 8/4/2016].

Schank, R. and Abelson, R. (1977): Scripts, Plans Goals and Understanding: An Inquiry Into Human Knowledge Structures. Hillsdale, NJ: Erlbaum. Shakespeare, William (2003): Hamlet, prince of denmark. Cambridge: Cambridge University Press.

Sicart, Miguel (2009): Ethics of Computer Games. Cambridge: The MIT Press.

Walsh, Richard (2011): Emergent Narrative in Interactive Media. NARRATIVE. (Vol. 19, num. 1, enero 2011). Ohio: Ohio State University Press. Wittgenstein, Ludwig (2004): Investigaciones filosóficas. Barcelona, España: Instituto de Investigaciones Filosóficas.

\section{Cómo citar este artículo en bibliografías - How to cite this article in bibliographies / references:}

ALBARRÁN, J. (2018): "La figura del ciberbardo y la experiencia interactiva del usuario en el diseño videolúdico: L.A. Noire". En Revista de la Asociación Española de Investigación de la Comunicación, vol. 5, número 9, pp. 17-24. 